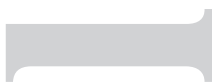

\title{
Higher-order and higher floating-point precision numerical approximations of finite strain elasticity moduli
}

\author{
Stephen John Connolly*, Donald Mackenzie, Yevgen Gorash
Department of Mechanical \& Aerospace Engineering, University of Strathclyde, Glasgow, GI IXJ, UK

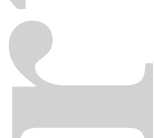

\section{SUMMARY}

Two real-domain numerical approximation methods for accurate computation of finite strain elasticity moduli are developed and their accuracy and computational efficiency investigated, with reference to hyperelastic constitutive models with known analytical solutions. The methods are higher-order and higher floating-point precision numerical approximation, the latter being novel in this context. A general formula for higher-order approximation finite difference schemes is derived and a new procedure is proposed to implement increased floating-point precision. The accuracy of the approximated elasticity moduli is investigated numerically using higher-order approximations in standard double precision and increased quadruple precision. It is found that as the order of the approximation increases, the elasticity moduli tend towards the analytical solution. Using higher floating-point precision, the approximated elasticity moduli for all orders of approximation are found to be more accurate than the standard double precision evaluation of the analytical moduli. Application of the techniques to a finite element problem shows that the numerically approximated methods obtain convergence equivalent to the analytical method but require greater computational effort. It is concluded that numerical approximation of elasticity moduli is a powerful and effective means of implementing advanced constitutive models in the finite element method without prior derivation of difficult analytical solutions. Copyright (c) 2019 John Wiley \& Sons, Ltd.

Received...

KEY WORDS: elasticity moduli, numerical differentiation, higher-order approximation, higher floatingpoint precision, hyperelasticity, nonlinear finite element method

To implement constitutive models within an implicit finite element method, both the stress tensor and consistent tangent moduli require computation. While stress computations determine the physical nature of the simulated component, tangent moduli are generally required only to ensure convergence and enable detection of stiffness instabilities [1]. For complicated constitutive models, such as when inelastic effects (viscoelasticity, plasticity or damage) are considered, derivation and implementation of analytical solutions for tangent moduli can be difficult and possibly intractable [2]. However, given that analytical tangent moduli are the linearized derivative of the stress tensor with respect to the strain tensor, alternative differentiation methods may be applied. Using these methods, the user may more easily implement existing constitutive models, or develop

\footnotetext{
*Correspondence to: Stephen John Connolly. Email: stephen.connolly@strath.ac.uk

This article has been accepted for publication and undergone full peer review but has not been through the copyediting, typesetting, pagination and proofreading process, which may lead to differences between this version and the Version of Record. Please cite this article as doi: 10.1002/nme.6176
} 
new ones, within the finite element method using only the stress relationships of a given constitutive model.

Alternative differentiation methods previously applied to obtain tangent moduli can be categorised into symbolic, automatic and numerical methods. In a symbolic differentiation method [3], algebraic mathematics software is used, or developed, such that the stress equations are differentiated symbolically to give the analytical expressions. Following this, the program codifies these expressions for numerical usage in the form required by the finite element solver. In automatic differentiation methods $[4,5,6,7]$, the derivative is obtained numerically by algorithmic accumulation of elementary evaluations related to the derivative. Perhaps due to the additional programming required, these methods are generally less common. A more prevalent approach applies numerical differentiation methods to obtain approximated tangent moduli, as proposed by Miehe [2].

For both Lagrangian and Eulerian finite strain configurations, Miehe [2] applied a perturbation technique wherein the stress components are perturbed in the six unique directions and orientations of the symmetric stress tensor. The tangent moduli are subsequently found by connection of the perturbed and stationary (unperturbed) stress components by the well-known forward difference formula. This method was further developed by Sun et al [8], adapting the expressions to the Jaumann-rate of the Cauchy stress, for implementation in the finite element software Abaqus. In these methods, the accuracy of the numerically approximated tangent moduli is dependent on the magnitude of the applied perturbation. Ideally, as the perturbation tends towards zero, the truncation error would also tend towards zero. However, due to finite floating point precision in these computational problems, the accuracy is limited due to a conflicting source of error: subtractive round-off error $[9,10]$.

The forward difference formula is a first-order approximation but higher-order approximations may also be utilised in this context. The central difference method is a second-order approximation that has been investigated in several studies $[11,12,13,14,15]$. In $[11,13,14,15]$, it is shown that second-order approximations give more accurate tangent moduli; but their optimal perturbation magnitude is larger due to the influence of the subtractive round-off error. Kiran and Khandelwal [14] use numerical approximations of tangent moduli up to the eighth-order. From[14], it is known that the computational cost increases as the order of approximation increases but the optimal perturbation magnitude and the numerical accuracy of higher-order approximation methods beyond second-order have not been investigated.

Approximation methods utilising complex numbers allow for smaller perturbation magnitudes and reduce truncation error, as they are not subject to subtractive round-off error. Complex approximation methods were first implemented for small strain problems by Prez-Foguet et al [11,12], using the complex step derivative approximation [16]. This method has also been applied to finite strain problems $[1,13]$. The numerically approximated Cauchy integral, implemented by Kiran and Khandelwal [14], is a further example of a complex numerical differentiation method used to compute tangent moduli. Another complex numerical differentiation method uses hyperdual numbers [17], applied in the context of finite elasticity, by Tanaka et al [18], for first and second derivatives of hyperelastic strain energy functions. The complex approximation methods allow for tangent moduli closer to the analytical solutions. However, implementation is more difficult than in the real domain. It requires redefinition of pre-programmed mathematical functions to allow for complex variables, otherwise its benefits are limited [11]. Such a change is not possible in older programming languages used in present commercial finite element software.

In this paper, two numerical approximation methods for accurate computation of elasticity moduli are developed and investigated, using approximation in the real-domain only. The methods are higher-order approximation and higher floating-point precision. As mentioned, higher-order approximations have been applied previously [14]; here they are investigated in detail to quantify the extent to which the truncation error can be reduced. A new procedure to utilise higher floating-point precision, which is novel in the context of computational finite strain tangent moduli, is proposed. In other applications, this has been shown to reduce round-off error[19]. The two methods are expected

This article is protected by copyright. All rights reserved. 
to be complementary, such that both truncation and round-off errors can be reduced to provide accurate numerical approximations of elasticity moduli.

The developed numerical approximation methods are investigated in terms of their numerical accuracy and computational efficiency. Although, as previously stated, the primary advantage of a numerical approximation method is when analytical solutions are intractable, the effectiveness of the method can only be investigated if compared with known analytical solutions. For this reason, the proposed methods are validated and compared for two hyperelastic constitutive models with known analytical solutions. Accuracy is assessed in both the Lagrangian and Eulerian configurations, using Fortran programs created for this purpose. In these programs, higher-order approximations of the elasticity moduli are compared to the analytical solutions using standard double precision and increased floating-point precision. Finite element analysis of a standard benchmark problem, using Abaqus/Standard, is employed to investigate computational efficiency, with Fortran user-subroutines for all numerical approximation methods. The Fortran programs and subroutines developed are openly available in a dataset [20].

General formulae for $n^{\text {th }}$-order approximations in Lagrangian and Eulerian configurations and the procedure to enable higher floating-point precision, are detailed in section 2 . The effect that higher-order approximations and higher floating-point precision have on the optimal perturbation magnitude and the minimum attainable error are discussed. A numerical investigation of the two methods is presented in section 3 for two hyperelastic constitutive models. Approximate elasticity moduli are evaluated and compared to analytically derived moduli in both material and spatial configurations. In section 4 the approximation methods are implemented and investigated within the finite element method, where the convergence behaviour and computational efficiency are assessed by comparison to the analytical solution. Conclusions are presented in section 5 .

\section{NUMERICAL APPROXIMATIONS OF ELASTICITY MODULI}

Here, the fundamental kinematic relationships of finite elasticity are defined with respect to Cartesian coordinates and base vectors are omitted. The fixed reference configuration $\Omega_{0}$ and current configuration $\Omega$ of a body are both defined in three-dimensional real coordinate space $\mathbb{R}^{3}$. A reference, or material, point $\mathbf{X} \in \Omega_{0}$ is mapped to its current, or spatial, equivalent point $\mathbf{x} \in \Omega$ by the deformation map $\chi$, where $\chi: \Omega_{0} \rightarrow \mathbb{R}^{3}$, such that $\mathbf{x}=\chi(\mathbf{X}, t)$, and $t \in \mathbb{R}$ is the time difference from the reference to the current configuration. Subsequent arguments are omitted here for brevity. The two-point deformation gradient tensor $\mathbf{F}$ may then be defined as $\mathbf{F}=\partial \chi / \partial \mathbf{X}$ or $\mathrm{F}_{a A}=\partial \chi_{a} / \partial \mathbf{X}_{A}$. The local volume ratio is given by $J=\operatorname{det}(\mathbf{F})$, where $J>0$ due to the impenetrability of matter. The symmetric right and left Cauchy-Green finite strain tensors are used throughout as the Lagrangian and Eulerian strain measures, respectively, given by $\mathbf{C}=\mathbf{F}^{\mathrm{T}} \mathbf{F}$ and $\mathbf{b}=\mathbf{F F}^{\mathrm{T}}$.

\subsection{Material elasticity tensor}

The material elasticity tensor is defined here without proof (see Miehe[2] and the references therein for details). The $2^{\text {nd }}$ Piola-Kirchhoff stress $\mathbf{S}$ is taken as a function of the right Cauchy-Green tensor C. As in the analytical derivation of the linearized material elasticity tensor[21], the linear relationship of the stress increment $\boldsymbol{\Delta} \mathbf{S}$ and the strain increment $\boldsymbol{\Delta} \mathbf{C}$ is defined as

$$
\boldsymbol{\Delta} \mathbf{S}=2 \frac{\partial \mathbf{S}}{\partial \mathbf{C}}: \frac{1}{2} \boldsymbol{\Delta} \mathbf{C}
$$

In (1), the term $2 \frac{\partial \mathbf{S}}{\partial \mathbf{C}}$ is the $4^{\text {th }}$-order material elasticity tensor $\mathbb{C}$. This implies that $\mathbb{C}$ may also be defined by numerical differentiation of the $2^{\text {nd }}$ Piola-Kirchhoff stress $\mathbf{S}$ with respect to the right Cauchy-Green tensor C. Here, only the first-order forward approximation is shown. The general formula of a first-order forward difference approximation is given by

$$
f^{\prime}(x)=\frac{f(x+\epsilon)-f(x)}{\epsilon}+\mathcal{O}(\epsilon)
$$

This article is protected by copyright. All rights reserved. 
where $\epsilon$ is a small perturbation magnitude, $\mathcal{O}(\epsilon)$ is the truncation error and $\mathcal{O}$ is the big $\mathcal{O}$ Landau symbol. Adapting this to compute the material elasticity moduli in the form proposed by Miehe[2]

$$
\mathbb{C}^{I J(K L)} \approx \frac{1}{\epsilon}\left[\hat{S}^{I J}\left(\hat{\mathbf{F}}_{(K L)}\right)-S^{I J}\right]
$$

Here, $\hat{S}^{I J}$ represents the perturbed stress tensors which are each a function of the perturbed right Cauchy-Green tensors and may otherwise be defined as an argument of the perturbed deformation gradient $\hat{\mathbf{F}}_{(K L)}$. The indices $I J$ are the components of the $2^{\text {nd }}$ Piola-Kirchhoff stress tensors and $K L$ indicate the direction and orientation of the applied perturbations. To compute the forward perturbed strains, and subsequently the perturbed stresses, the perturbed deformation gradients are calculated by

$$
\hat{\mathbf{F}}_{(K L)}=\mathbf{F}+\Delta \hat{\mathbf{F}}_{(K L)}
$$

The applied perturbation, denoted as $\boldsymbol{\Delta} \hat{\mathbf{F}}_{(K L)}$, is defined as

$$
\Delta \hat{\mathbf{F}}_{(K L)}=\frac{\epsilon}{2}\left(\mathbf{F}^{-\mathrm{T}} E_{K} \otimes E_{L}+\mathbf{F}^{-\mathrm{T}} E_{L} \otimes E_{K}\right)
$$

Including the indices of the deformation gradient in (4) and using the Kronecker delta $\delta_{i j}$, the perturbed deformation gradients are calculated using

$$
\left(\hat{F}_{(K L)}\right)_{i J}=F_{i J}+\frac{\epsilon}{2}\left(F_{i K}{ }^{-\mathrm{T}} \delta_{L J}+F_{i L}{ }^{-\mathrm{T}} \delta_{K J}\right)
$$

The perturbed stresses are calculated, allowing calculation of the approximated material elasticity moduli using (3). Noting the symmetry of both the stress and strain definitions used, the elasticity tensor (3) has minor symmetries such that: $\mathbb{C}^{I J K L}=\mathbb{C}^{J I K L}=\mathbb{C}^{I J L K}$. It may therefore be computed using only six approximations, or four if the problem is reduced to assume $2 \mathrm{D}$ plane strain or axisymmetric conditions. Voigt notation is employed with indices $(1,2,3,4,5,6)$ representing $(11,22,33,12,13,23)$, respectively. Using the indices $A=I J$ and $B=K L$ (in $3 \mathrm{D} \mathrm{A}$ and $\mathrm{B}=1$, $2,3,4,5,6$; in $2 \mathrm{D} \mathrm{A}$ and $\mathrm{B}=1,2,3,4)$ the approximated material elasticity tensor (3) is given in the convenient form

$$
\mathbb{C}^{A(B)} \approx \frac{1}{\epsilon}\left[\hat{S}^{A}\left(\hat{\mathbf{F}}_{(B)}\right)-S^{A}\right]
$$

\subsection{Spatial elasticity tensor}

For computation of the approximate spatial elasticity tensor, the Kirchhoff stress tensor $\tau$ and the left Cauchy-Green tensor $\mathbf{b}$ are used. The spatial elasticity tensor is derived as the Jaumann-rate of the Cauchy stress, which is the form required by Abaqus/Standard. The motivation for this framework is due to the later use of Abaqus/Standard for validation and investigations using the finite element method. Although, conversions and corrections from this framework to alternative spatial tangent moduli are well-known [22]. Proofs are omitted and only the first-order forward approximation is given.

Expanding the increment of the strain tensor $\Delta \mathbf{C}=\left(\mathbf{F}^{\mathrm{T}} \boldsymbol{\Delta} \mathbf{F}+\boldsymbol{\Delta} \mathbf{F}^{\mathrm{T}} \mathbf{F}\right)$ followed by a push-forward operation, the spatial equivalent of (1) is given as

$$
\Delta \boldsymbol{\tau}-\boldsymbol{\tau}\left(\boldsymbol{\Delta} \mathbf{F} \mathbf{F}^{-1}\right)-\boldsymbol{\tau}\left(\boldsymbol{\Delta} \mathbf{F} \mathbf{F}^{-1}\right)^{\mathrm{T}}=: \frac{1}{2}\left[\left(\boldsymbol{\Delta} \mathbf{F} \mathbf{F}^{-1}\right)+\left(\boldsymbol{\Delta} \mathbf{F F}^{-1}\right)^{\mathrm{T}}\right]
$$

The $4^{\text {th }}$-order tensor denotes the spatial elasticity tensor, which is further defined in terms of the Oldroyd-rate, or Lie time derivative, of the Kirchhoff stress $\mathcal{L}_{v}(\boldsymbol{\tau})$, and the rate of deformation tensor $\mathbf{d}$ as $\mathcal{L}_{v}(\boldsymbol{\tau})=: \mathbf{d}$. The rate of deformation has its usual definition in terms of the spatial velocity tensor $\mathbf{L}$, which in turn is defined in terms of the rate of the deformation gradient, as follows $\mathbf{d}=\frac{1}{2}\left(\mathbf{L}-\mathbf{L}^{\mathrm{T}}\right)=\frac{1}{2}\left(\dot{\mathbf{F}} \mathbf{F}^{-1}-\left(\dot{\mathbf{F}} \mathbf{F}^{-1}\right)^{\mathrm{T}}\right)$. The derivatives may be linearized[21] and can therefore also be numerically approximated. However, unlike the material tensor, geometric contributions are

This article is protected by copyright. All rights reserved. 
present to preserve objectivity. The first-order forward approximation of the spatial elasticity tensor is therefore defined as

$$
{ }^{i j}(k l) \approx \frac{1}{\epsilon}\left[\hat{\tau}^{i j}\left(\hat{\mathbf{F}}_{(k l)}\right)-\tau^{i j}\right]-(\mathbf{1} \odot \boldsymbol{\tau}+\boldsymbol{\tau} \odot \mathbf{1})^{i j(k l)}
$$

where $\hat{\tau}^{i j}$ represents the perturbed Kirchhoff stress tensors, which are a function of the spatial perturbed deformation gradients $\hat{\mathbf{F}}_{(k l)}, \mathbf{1}$ is the $2^{\text {nd }}$-order identity tensor and the symbol $\odot$ has been introduced for convenience, as in[21], to signify the $4^{\text {th }}$-order product of two $2^{\text {nd }}$-order tensors:

$$
(\mathbf{A} \odot \mathbf{B})_{i j k l}=\frac{1}{2}\left(A_{i k} B_{j l}+A_{i l} B_{j k}\right)
$$

Equivalent to the material implementation, the spatial perturbed deformation gradient is given by

$$
\hat{\mathbf{F}}_{(k l)}=\mathbf{F}+\Delta \hat{\mathbf{F}}_{(k l)}
$$

However, the spatial perturbed deformation gradients are calculated using

$$
\Delta \hat{\mathbf{F}}_{(k l)}=\frac{\epsilon}{2}\left(e_{k} \otimes e_{l} \mathbf{F}+e_{l} \otimes e_{k} \mathbf{F}\right)
$$

Then including the indices and the Kronecker delta in (11), the perturbed deformation gradients are calculated by

$$
\left(\hat{F}_{(k l)}\right)_{i J}=F_{i J}+\frac{\epsilon}{2}\left(\delta_{i k} F_{l J}+\delta_{i l} F_{k J}\right)
$$

In Voigt notation, with the same interpretations as in the material tensor, the spatial elasticity tensor defined in terms of the Oldroyd-rate of the Kirchhoff stress is simplified to

$$
{ }^{a(b)} \approx \frac{1}{\epsilon}\left[\hat{\tau}^{a}\left(\hat{\mathbf{F}}_{(b)}\right)-\tau^{a}\right]-(\mathbf{1} \odot \boldsymbol{\tau}+\boldsymbol{\tau} \odot \mathbf{1})^{a(b)}
$$

Some commercial finite element solvers require the spatial elasticity tensor to be defined in terms of the Jaumann-rate of the Cauchy stress, denoted here as $A B Q$. The Oldroyd-rate of the Kirchhoff stress and the Jaumann-rate of the Cauchy stress are related[23] by the following

$$
A B Q^{i j k l}=\frac{1}{J}\left[i j k l+(\mathbf{1} \odot \boldsymbol{\tau}+\boldsymbol{\tau} \odot \mathbf{1})^{i j k l}\right]
$$

Then by substitution of (9) the geometric contributions cancel to give the Jaumann-rate of the Cauchy stress in the compact form

$$
A B Q^{i j(k l)} \approx \frac{1}{J \epsilon}\left[\hat{\tau}^{i j}\left(\hat{\mathbf{F}}_{(k l)}\right)-\tau^{i j}\right]
$$

And expressed in Voigt notation it is given as

$$
A B Q{ }^{a(b)} \approx \frac{1}{J \epsilon}\left[\hat{\tau}^{a}\left(\hat{\mathbf{F}}_{(b)}\right)-\tau^{a}\right]
$$

\subsection{Higher-order numerical approximations}

General equations are derived for higher-order approximations of both the material and spatial elasticity moduli. While higher-order approximations of forward and backward difference methods are possible, only central difference methods are investigated in detail, as they are generally of higher numerical accuracy[24]. Furthermore, central difference methods are more convenient for investigation as their truncation error exponentially decreases twofold compared with forward and backward methods: $\mathcal{O}\left(\epsilon^{2 n}\right)$ compared with $\mathcal{O}\left(\epsilon^{n}\right)$. For clarity, central difference schemes are referred to by their order of approximation. For example, the first central difference is a secondorder approximation and so is referred to as the second-order central difference approximation.

This article is protected by copyright. All rights reserved. 
For a fixed perturbation magnitude, increasing the order of the approximation will, in theory, reduce the truncation error. For this, the higher-order approximations require more forward and backward perturbed points. In the central difference approximations applied here, an equal number of equidistant forward and backward perturbations are used. To ensure that the approximation is found at the centre point, weighted coefficients are prescribed. The general formula for a central difference of $n^{\text {th }}$-order, where $n$ is an even number, from[25], is given as

$$
f^{\prime}(x)=\left(\frac{1}{\epsilon} \sum_{2 m=-n_{\text {even }}}^{n_{\text {even }}}\left(c_{m}\right) f\left(x_{m}\right)\right)+\mathcal{O}\left(\epsilon^{2 \mathrm{n}}\right)
$$

Here, $m$ refers to the position of the approximation as if it were to lie on a linear grid, i.e. $m=-2$ is a twice backward perturbed point, which is non-zero for central difference schemes of fourthorder and greater. Then the argument $\left(x_{m}\right)=(x+m \epsilon)$ is calculated. For forward and backward perturbed points, the coefficients $c_{m}$ are calculated by the equation

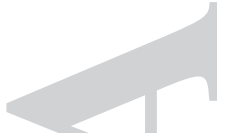

$$
c_{m}=\frac{(-1)^{m+1}\left[\left(\frac{n}{2}\right) !\right]^{2}}{m\left(\frac{n}{2}-m\right) !\left(\frac{n}{2}+m\right) !}
$$

Manual calculation of the required coefficients can be tedious and so an algorithm similar to that of Fornberg[26] may be used to obtain them. The coefficients for the central difference schemes used in this study, up to twelfth-order, are given in Table II in Appendix A.

2.3.1. Higher-order approximations of material elasticity moduli Higher-order approximations of the material elasticity moduli require calculation of additional perturbed $2^{\text {nd }}$ Piola-Kirchhoff stresses. The stresses are a function of the perturbed deformation gradients, which are therefore calculated first using a modification of (4)

$$
\overbrace{\left(\hat{\mathbf{F}}_{(K L)}\right)}^{m}=\mathbf{F}+m\left(\boldsymbol{\Delta} \hat{\mathbf{F}}_{(K L)}\right)
$$

The notation $\overbrace{(\cdot)}^{m}$ is introduced to identify the position, $m$, of the approximation, with $m$ defined as previous. In 3D the $6 n$ ( $4 n$ for $2 \mathrm{D}$ ) perturbed $2^{\text {nd }}$ Piola-Kirchhoff stresses are then calculated. The general formula for central difference approximate elasticity moduli is found by combining (3) and (18) to give

$$
\mathbb{C}^{I J(K L)} \approx\left[\frac{1}{\epsilon}(\sum_{2 m=-n_{\text {even }}}^{n_{\text {even }}}\left(c_{m}\right) \hat{S}^{I J} \overbrace{\left(\hat{\mathbf{F}}_{(K L)}\right)}^{m})\right]+\mathcal{O}\left(\epsilon^{2 n}\right)
$$

In Voigt notation, where $A$ and $B$ are as previously defined, this is defined as

$$
\mathbb{C}^{A(B)} \approx\left[\frac{1}{\epsilon}(\sum_{2 m=-n_{\text {even }}}^{n_{\text {even }}}\left(c_{m}\right) \hat{S}^{A} \overbrace{\left(\hat{\mathbf{F}}_{(B)}\right)^{A}}^{m})\right]+\mathcal{O}\left(\epsilon^{2 n}\right)
$$

2.3.2. Higher-order approximations of material elasticity moduli The higher-order approximations are then modified equivalently to give the corresponding equations for the spatial elasticity tensor. In the spatial configuration, the perturbed deformation gradients are calculated using

$$
\overbrace{\left(\hat{\mathbf{F}}_{(k l)}\right)}^{m}=\mathbf{F}+m\left(\Delta \hat{\mathbf{F}}_{(k l)}\right)
$$

This article is protected by copyright. All rights reserved. 
The general equation for the spatial elasticity tensor in terms of the Oldroyd-rate of the Kirchhoff stress is given by

$$
i j(k l) \approx\left[\frac{1}{\epsilon}(\sum_{2 m=-n_{\text {even }}}^{n_{\text {even }}}\left(c_{m}\right) \hat{\tau}^{i j} \overbrace{\left(\hat{\mathbf{F}}_{(k l)}\right)}^{m})-(\mathbf{1} \odot \boldsymbol{\tau}+\boldsymbol{\tau} \odot \mathbf{1})^{i j(k l)}\right]+\mathcal{O}\left(\epsilon^{2 n}\right)
$$

And in Voigt notation is defined

$$
\operatorname{la}^{a(b)} \approx\left[\frac{1}{\epsilon}(\sum_{2 m=-n_{\text {even }}}^{n_{\text {even }}}\left(c_{m}\right) \hat{\tau}^{a} \overbrace{\left(\hat{\mathbf{F}}_{(k l)}\right)}^{m})-(\mathbf{1} \odot \boldsymbol{\tau}+\boldsymbol{\tau} \odot \mathbf{1})^{a(b)}\right]+\mathcal{O}\left(\epsilon^{2 n}\right)
$$

As in (16), the geometric contributions cancel to give the general formula for the spatial elasticity tensor in terms of the Jaumann-rate of the Cauchy stress as

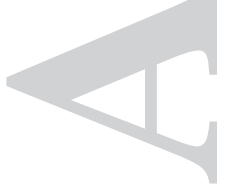

$$
i j(k l) \approx\left[\frac{1}{J \epsilon}(\sum_{2 m=-n_{\text {even }}}^{n_{\text {even }}}\left(c_{m}\right) \hat{\tau}^{i j} \overbrace{\left(\hat{\mathbf{F}}_{(k l)}\right)}^{m})\right]+\mathcal{O}\left(\epsilon^{2 n}\right)
$$

And in Voigt notation it is given by

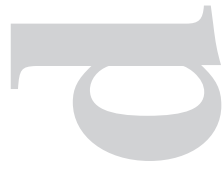

$$
a(b) \approx\left[\frac{1}{J \epsilon}(\sum_{2 m=-n_{\text {even }}}^{n_{\text {even }}}\left(c_{m}\right) \hat{\tau}^{a} \overbrace{\left(\hat{\mathbf{F}}_{(b)}\right)}^{m})\right]+\mathcal{O}\left(\epsilon^{2 n}\right)
$$

\subsection{Higher floating-point precision}

While higher-order approximations increase the exponential decay of the truncation error, by $\mathcal{O}\left(\epsilon^{2 n}\right)$ for increasing $n$, the benefit of this is well-known to be restricted by the conflicting round-off errors[9, 27]. However, with higher floating-point precision, the range of perturbation magnitudes for which round-off error is negligible may increase. Due to its availability within current Fortran compilers, quadruple precision is used. In Fortran, all arrays, variables and constants are initially defined in quadruple precision. The variables and arrays required by the finite element solver are also defined equivalently in double precision, which are the stress and elasticity tensors, and any other calculated values of interest. After computation of the quadruple precision arrays, the values are returned to double precision by equating these arrays to their double precision counterparts. This truncates the higher precision values to approximately 16 significant figures. The method has not previously been implemented or investigated in this context. For all orders of approximation, quadruple precision enables twice the number of significant figures allowing smaller perturbation magnitudes. This is expected to result in lower truncation error and more accurate approximations of the elasticity moduli.

\subsection{Optimal perturbation magnitude}

The optimal perturbation magnitude is that which exactly minimises the impact of both truncation and round-off errors. As these are known to vary for even a scalar function of an independent scalar variable, determining the exact optimal perturbation magnitude is generally not possible. An approximation of the optimal perturbation can be obtained by optimisation [10], which, in this context, would require a minimisation in which all tensor components are considered. A simpler approach by Miehe[2] concludes that for a first-order approximation of the first derivative, the optimal perturbation $\epsilon^{o p t}$ may be approximated as

$$
\epsilon^{o p t} \approx \sqrt{\text { macheps }}
$$

This article is protected by copyright. All rights reserved. 
The machine epsilon, macheps is the smallest computable $\kappa$ for $1+\kappa>1$ to hold true. The approximation (28) can be seen to hold true graphically in log-log plots of relative error vs perturbation magnitude from later studies[1, 11, 13, 14, 18]. Based on (28) and the observations of Prez-Foguet et al[11], a simple general equation for higher-order derivatives and higher-order approximations is proposed here as

$$
\epsilon^{o p t} \approx(\text { macheps })^{\frac{1}{n+d}}
$$

The superscripts $n$ and $d$ are the orders of the approximation and of the derivative, respectively. The equation draws on the observation that the total error corresponds to the truncation error: Error $\approx \gamma \epsilon^{n}$, where $\gamma$ is a constant, until it intercepts the round-off errors. The round-off errors then increase the total error by Error $\approx \omega \epsilon^{-d}$, where $\omega$ is a constant. Therefore, by assuming the minimum error occurs when truncation and round-off errors are equal, as in Prez-Foguet et al[11], and that the constants are a function of macheps such that (28) is satisfied, the optimal perturbation magnitude may be approximated by (29).

This equation permits an approximation of the optimal perturbation magnitude, which can be seen to hold true to the error plots of previous studies and is in agreement with (28). Comparisons with previous studies $[1,11,13,14,18]$ is not a complete validation as these approximations only investigated up to second-order approximations and second-order derivatives. The validity for firstorder derivatives and approximations of up to the twelfth-order is assessed in section 3.3.

The proposed equation (29) predicts that for increasingly higher-order approximations the optimal perturbation size exponentially increases due to conflicting round-off error. It also predicts that the use of quadruple precision will lower the optimal perturbation magnitude. For example, a first-order forward approximation in double precision (macheps $=10^{-16}, n=1$ and $d=1$ ) the optimal perturbation magnitude is calculated as $\epsilon^{o p t} \approx 10^{-8}$. With the same approximation scheme in quadruple precision (macheps $=10^{-32}, n=1$ and $d=1$ ) the optimal perturbation magnitude is now predicted as $\epsilon^{o p t} \approx 10^{-16}$. In terms of the truncation error $\mathcal{O}\left(\epsilon^{n}\right)$, the use of quadruple precision reduces the error by a squared power. Given that double precision numbers are stored with $10^{-16}$ significant figures, this predicts that a first-order approximation using quadruple precision will obtain solutions whose error is approximately equal to the finite precision limit. Following an investigation into higher-order approximations in double and quadruple precision, the optimal perturbation magnitude and the validity of equation (29) is discussed in further detail in the next section.

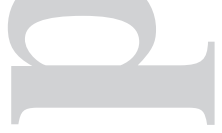

\section{NUMERICAL INVESTIGATION}

The use of higher-order numerical approximation methods is investigated in standard double precision and with the proposed quadruple precision method. The methods are assessed in terms of their numerical accuracy through comparison with known analytical solutions for both material and spatial configurations. Most materials include some aspect of inelasticity, such as viscoelasticity[28, 29, 30], plasticity[31], damage[32, 33] or a combination of these behaviours [34, 35]. However, only hyperelasticity is considered here for simple constitutive models with known analytical solutions. These allow numerical investigation and validation of the proposed numerical approximation methods.

\subsection{Constitutive hyperelasticity equations}

A hyperelastic constitutive equation may be phenomenologically or mechanistically derived, i.e. it defines a mathematical fit to macroscopic behaviours or is defined by equations describing the internal molecular mechanisms, respectively. Furthermore, the definition may be given in terms of one of several strain measures. Connecting all hyperelastic materials is the assumption that a Helmholtz strain energy function $\Psi(\cdot)$ exists, defined in terms of unit volume where the argument $(\cdot)$ represents any strain measure(s). Due to the difference of shear and bulk behaviours in hyperelastic materials, the deformation is split into isochoric $\overline{\mathbf{F}}$ and volumetric $J^{1 / 3} \mathbf{I}$ components:

This article is protected by copyright. All rights reserved. 
$\mathbf{F}=\left(J^{1 / 3} \mathbf{I}\right) \overline{\mathbf{F}}=J^{1 / 3} \overline{\mathbf{F}}$, which implies $\overline{\mathbf{C}}=J^{-2 / 3} \mathbf{C}$ and $\overline{\mathbf{b}}=J^{-2 / 3} \mathbf{b}$, where $J=\operatorname{det} \mathbf{F}$. The strainenergy function is similarly decoupled into incompressible isochoric and dilational volumetric components $\left.W()^{\circ}\right)$ and $U(J)$ such that $\Psi=W+U$. Stress and elasticity tensors are also decomposed into equivalent components.

Two hyperelastic constitutive models are used throughout: the neo-Hookean[36] and Gent[37] models. These are chosen due to their differences in numerical stability and function smoothness. The neo-Hookean model is unconditionally stable and a smooth function. The Gent model has a clearly defined stability limit, where the stiffness increases asymptotically as the strain tends towards the finite extensibility limit of the material. Stability here refers to the positive definiteness of stress and strain increments, as discussed by Hartmann[38] for hyperelastic parameter estimation.

The neo-Hookean and Gent strain energy functions are defined only in terms of an isochoric energy contribution. As an incompressible hybrid formulation is later used to enforce incompressibility, the volumetric contributions to the total energy, stress and elasticity moduli are disregarded. The isochoric energy of these models is defined in terms of the first principal invariant of the left or right Cauchy-Green strain tensor, $\bar{I}_{1}=\operatorname{tr}(\overline{\mathbf{C}})=\operatorname{tr}(\overline{\mathbf{b}})$. The isochoric strain energy functions for the neo-Hookean and Gent models, $W_{n H}$ and $W_{G}$ respectively, are defined by

$$
\begin{gathered}
W_{n H}=C_{1}\left(\bar{I}_{1}-3\right) \\
W_{G}=-\frac{\mu J_{m}}{2} \ln \left(1-\frac{\bar{I}_{1}-3}{J_{m}}\right)
\end{gathered}
$$

In these equations, $C_{1}$ and $\mu$ are parameters related to the shear stiffness and $J_{m}=\bar{I}_{1 m}-3$ is a function of the maximum first invariant $\bar{I}_{1 m}$, which defines an asymptotic limit when $\bar{I}_{1}$ tends towards $\bar{I}_{1 m}$. The parameters $C_{1}, \mu$ and $J_{m}$ are set to $0.5(\mathrm{MPa}), 1.0$ (MPa) and $22.5(-)$ respectively throughout.

An analytical implementation requires closed-form solutions for the elasticity moduli in both material and spatial configurations. The material stress and elasticity tensors, as in Steinmann et al[39] and Hossain and Steinmann[40], are respectively defined in terms of the $2^{\text {nd }}$ Piola-Kirchhoff stress tensor and the equivalent material elasticity tensor. The spatial stress and elasticity tensors, as defined by Kaliske and Rothert[41], are initially defined in terms of the Kirchhoff stress tensor and the Oldroyd-rate of the Kirchhoff stress respectively. They are then respectively adapted to be defined in terms of the Cauchy stress and Jaumann-rate of the Cauchy stress. The implementation used here is further simplified for strain energy functions given only in terms of the first CauchyGreen invariant. These are given in Appendix B, where the volumetric contributions are also included for completeness.

\subsection{Investigation of approximate elasticity moduli}

The accuracy of the approximate elasticity moduli is investigated by comparison to the analytical solutions. The average relative error is calculated for all (Voigt notation) tensor components using five unique deformation gradients. The deformation gradients are input to a Fortran program to calculate the stress tensor and elasticity moduli, these programs are available in the dataset[20]. Physically, these deformation gradients represent a homogeneously deformed element of material. The five deformation gradients may be respectively described as follows: undeformed, uniaxial tension, uniaxial compression, shear without dilation and shear with dilation. In matrix form, they are given as

$$
F_{1}=\left(\begin{array}{ccc}
1 & 0 & 0 \\
0 & 1 & 0 \\
0 & 0 & 1
\end{array}\right) ; F_{2}=\left(\begin{array}{ccc}
4.5 & 0 & 0 \\
0 & \frac{1}{\sqrt{4.5}} & 0 \\
0 & 0 & \frac{1}{\sqrt{4.5}}
\end{array}\right) ; F_{3}=\left(\begin{array}{ccc}
0.2 & 0 & 0 \\
0 & \frac{1}{\sqrt{0.2}} & 0 \\
0 & 0 & \frac{1}{\sqrt{0.2}}
\end{array}\right) ; F_{4}=\left(\begin{array}{ccc}
3 & 1 & 0 \\
1 & 1 & 0 \\
0 & 0 & 0.5
\end{array}\right) ; F_{5}=\left(\begin{array}{ccc}
1.1 & 0.2 & 0.2 \\
0 & 0.9535 & 0.2 \\
0 & 0 & 0.9535
\end{array}\right)
$$

The relative error is calculated for each approximation method using a range of perturbation magnitudes. The investigations use perturbations of $1 \times 10^{x}$ and $3 \times 10^{x}$, where $x$ is an integer, to provide a good insight into the range of suitable perturbation magnitudes and to find an approximate value of the optimal perturbation magnitude. For double precision, perturbation magnitudes from $10^{0}$ down to $10^{-18}$ are used. For quadruple precision, the range is extended from $10^{0}$ to $10^{-36}$.

This article is protected by copyright. All rights reserved. 
(1)

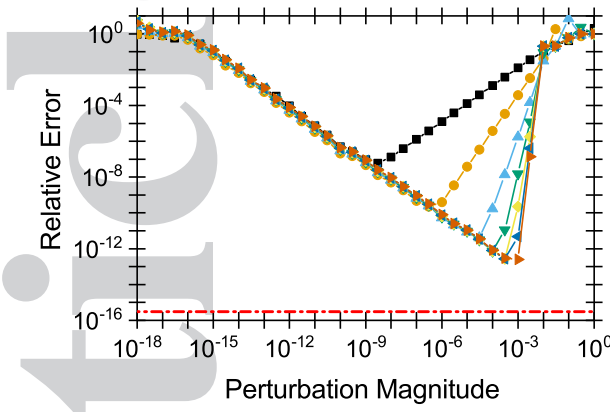

(a) neo-Hookean, double precision

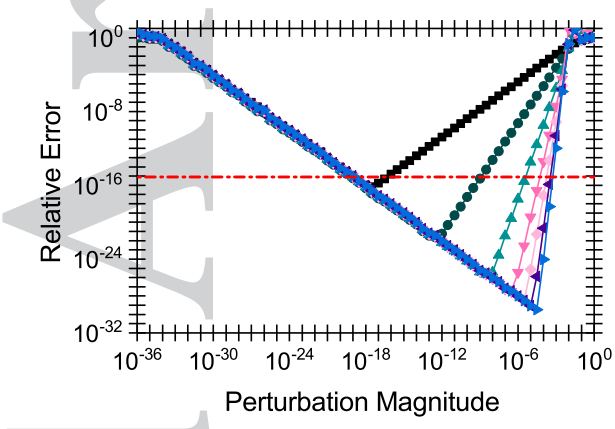

(c) neo-Hookean, quadruple precision

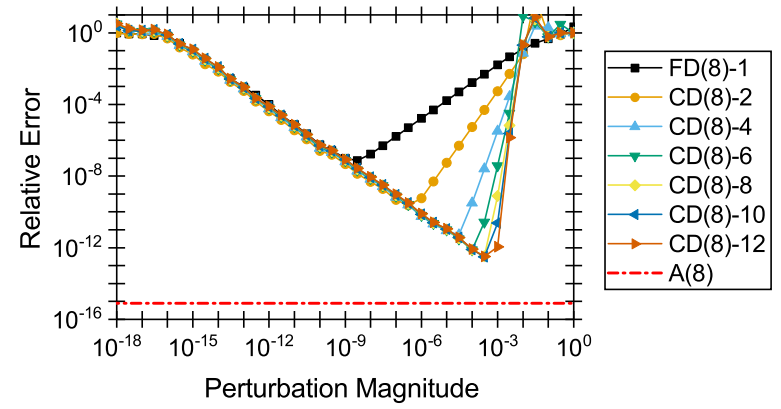

(b) Gent, double precision

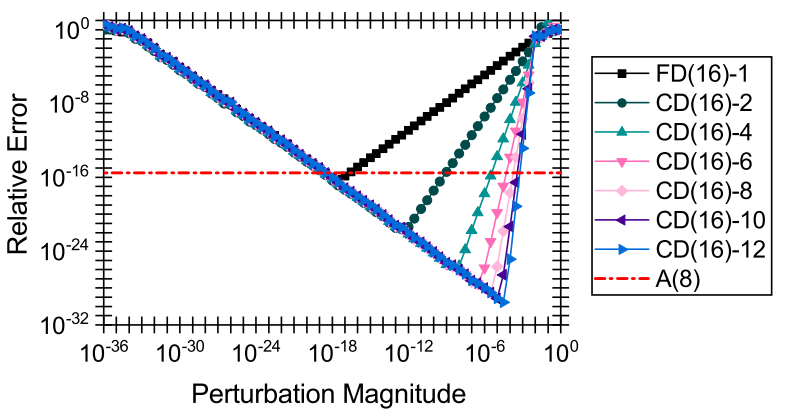

(d) Gent, quadruple precision

Figure 1. Relative error of material elasticity moduli approximations.

Perturbation magnitudes smaller than these ranges are much lower than macheps and thus result in an unperturbed deformation gradient for real domain approximations, i.e. $1+\epsilon>1$ is false. The relative error is calculated by comparison of the approximate moduli, $\mathbb{C}_{A p p}$. and App., to the quadruple precision analytical elasticity moduli, $\mathbb{C}_{A 16}$ and ${ }_{A 16}$, for the material and spatial configurations respectively. This also allows for the double precision analytical elasticity moduli, denoted $\mathrm{A}(8)$, to be included in the comparison. The error between the double and quadruple precision analytical moduli is a result of the truncation and rounding errors throughout its calculation. The relative error $E_{R}$ is calculated and averaged for each index of the elasticity tensor in Voigt notation by the following equation

$$
E_{R}=\left[\sum_{a, b=1}^{6}\left(\mathbb{C}_{A 16}{ }^{a b}-\mathbb{C}_{A p p .}{ }^{a b}\right)^{2}\right]^{\frac{1}{2}} /\left[\sum_{a, b=1}^{6}\left(\mathbb{C}_{A 16}{ }^{a b}\right)^{2}\right]^{\frac{1}{2}}
$$

To independently investigate the effect of higher-order approximations and higher floating-point precision, approximated material and spatial moduli are compared for both constitutive models using double and quadruple precision for approximations up to the twelfth-order. The results are plotted using log-log graphs of perturbation magnitude vs relative error. These plots also reveal the approximate optimal perturbation magnitude. In the following numerical investigations, the first two letters indicate the type of approximation scheme used (FD and CD are the forward and central differences respectively), the number in brackets is the precision used (double precision is (8) and quadruple precision is (16)), and the number following the hyphen indicates the order of the approximation.

3.2.1. Material elasticity moduli The result of the comparison between the approximated and analytical material elasticity moduli is shown for double precision in Figure 1a and 1b. These plots show there is little difference between the neo-Hookean and Gent models for all approximations, suggesting that the approximation methods are not affected by stability differences or function

This article is protected by copyright. All rights reserved. 


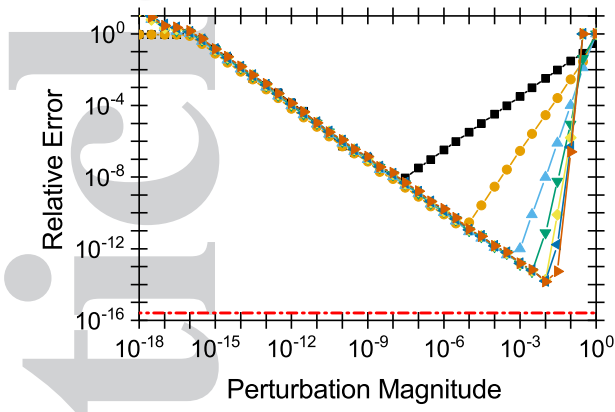

(a) neo-Hookean, double precision

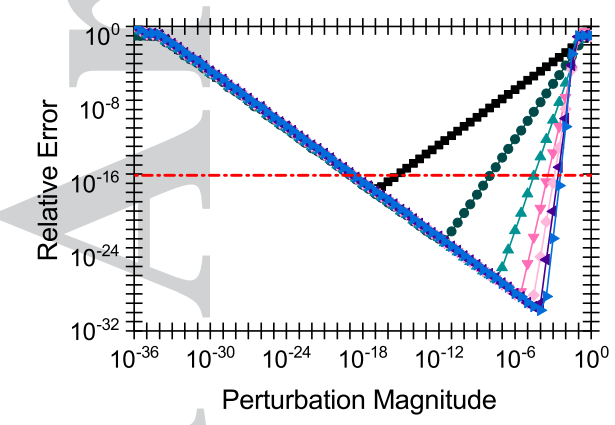

(c) neo-Hookean, quadruple precision

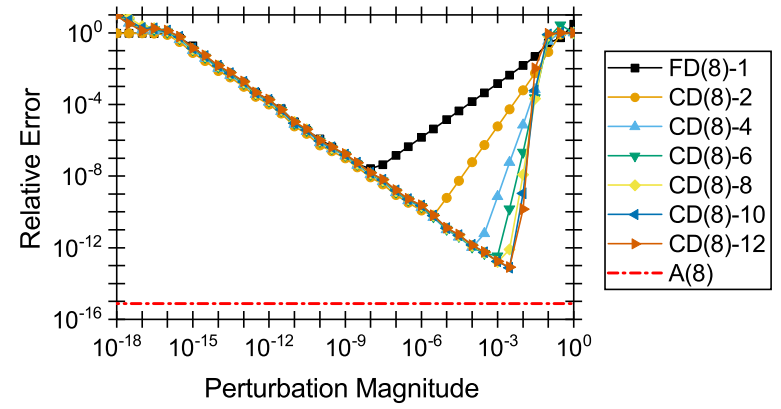

(b) Gent, double precision

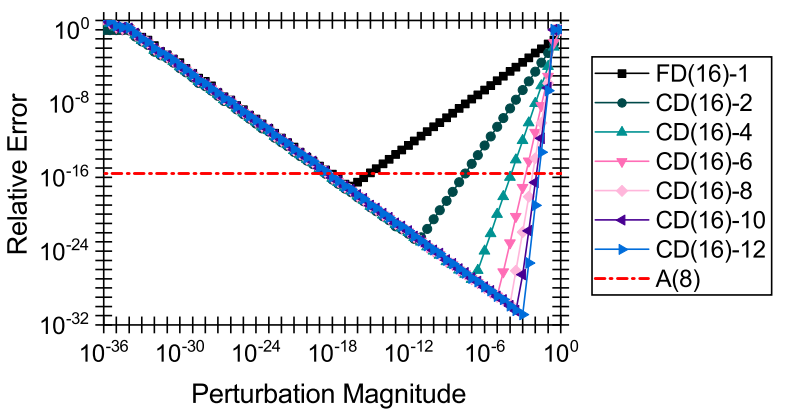

(d) Gent, quadruple precision

Figure 2. Relative error of spatial elasticity moduli approximations.

smoothness. For both constitutive models, lower relative error is obtained with an increased order of approximation but with diminishing returns. Once round-off errors are significant, as the perturbation magnitude decreases, all orders of approximation converge to the same error as the first-order approximation.

With quadruple precision, shown in Figure $1 \mathrm{c} \& 1 \mathrm{~d}$, the approximated elasticity moduli reach higher accuracy than the double precision analytical solution for all orders of approximation. When truncated to double precision, these quadruple precision approximations of the material elasticity moduli are generally identical to the double precision analytical solutions. This is true for all non-zero terms; otherwise, values close to zero may still have a residual value of around $10^{-16}$ since these are not lost in truncation. However, given their magnitude, these errors are physically negligible.

At larger perturbation magnitudes, $\geq 10^{-2}$, it is found that the relative error of the approximations does not follow the expected logarithmic gradient equal to the order of the approximation. Since this is present in both double and quadruple precision, this error is not due to truncation or rounding errors. Also, as it is present in both neo-Hookean and Gent constitutive models, it is not due to stability or smoothness. The error is therefore due to invalid truncation error [10]. Approximations greater than first-order result in an invalid perturbation, which restricts the accuracy of higher-order approximations whose optimal perturbation is expected to occur within this range.

3.2.2. Spatial elasticity moduli The investigation of the approximated spatial elasticity moduli uses the Jaumann-rate of the Cauchy stress. The results are shown in Figure 2 for both neo-Hookean and Gent constitutive models. These results are similar to those of the material elasticity moduli but the spatial approximations are less affected by invalid perturbation error. In double precision, the increased order of approximation generally achieves lower error. However, approximations of greater than the eighth-order show diminishing increases in accuracy. The quadruple precision results are similar to those of the material elasticity moduli, though their error continues to decrease for higher-order approximations since the optimal perturbation occurs at lower (valid) perturbation 
magnitudes. As with the material elasticity moduli, the use of higher floating-point precision obtains more accurate elasticity moduli than the double precision analytical solution for all orders of approximation.

\subsection{Optimal perturbation magnitude}

The approximate optimal perturbation magnitudes are calculated using (29) for both double and quadruple precision and all orders of approximations used in the previous section. In Table I the approximations are compared to the measured spatial neo-Hookean optimal perturbation magnitudes, where measured values are taken to the nearest magnitude. The approximate values of (29) are generally reasonable for lower orders of approximation; but the optimal perturbation magnitudes are not in agreement for double precision higher-order approximations due to invalid perturbation error.

The nature of truncation and round-off errors are examined further using more perturbation magnitudes around the minimum error. The neo-Hookean spatial elasticity moduli are approximated using fourth- and eighth-order central difference approximations in quadruple precision with 450 linear steps of the perturbation magnitude for each order of magnitude, shown in Figure 3. Using more points reveals that the truncation errors steadily decrease at a logarithmic gradient equal to the order of the approximation. However, when approaching the optimal perturbation magnitude, the round-off errors fluctuate, as highlighted in Figure 3. Following the optimal perturbation, they continue to fluctuate but tend towards an indirectly proportional relationship between the perturbation magnitude and the error. These results show further agreement with equation (29) and the related discussion of section 2.5 .

Table I. Approximate optimal perturbation magnitudes, from equation (29) and Figure 2a and 2c.

\begin{tabular}{lccccccc}
\hline & FD-1 & CD-2 & CD-4 & CD-6 & CD-8 & CD-10 & CD-12 \\
\hline Double: calculated & $1.0 E^{-8}$ & $4.6 E^{-6}$ & $6.3 E^{-4}$ & $5.2 E^{-3}$ & $1.7 E^{-2}$ & $3.5 E^{-2}$ & $5.9 E^{-2}$ \\
Double: measured & $1.0 E^{-8}$ & $1.0 E^{-5}$ & $1.0 E^{-3}$ & $1.0 E^{-3}$ & $1.0 E^{-2}$ & $1.0 E^{-2}$ & $1.0 E^{-2}$ \\
Quadruple: calculated & $1.0 E^{-16}$ & $2.2 E^{-11}$ & $4.1 E^{-7}$ & $2.7 E^{-5}$ & $2.8 E^{-4}$ & $1.2 E^{-3}$ & $3.5 E^{-3}$ \\
Quadruple: measured & $1.0 E^{-17}$ & $1.0 E^{-11}$ & $1.0 E^{-7}$ & $1.0 E^{-5}$ & $1.0 E^{-4}$ & $1.0 E^{-4}$ & $1.0 E^{-3}$ \\
\hline
\end{tabular}
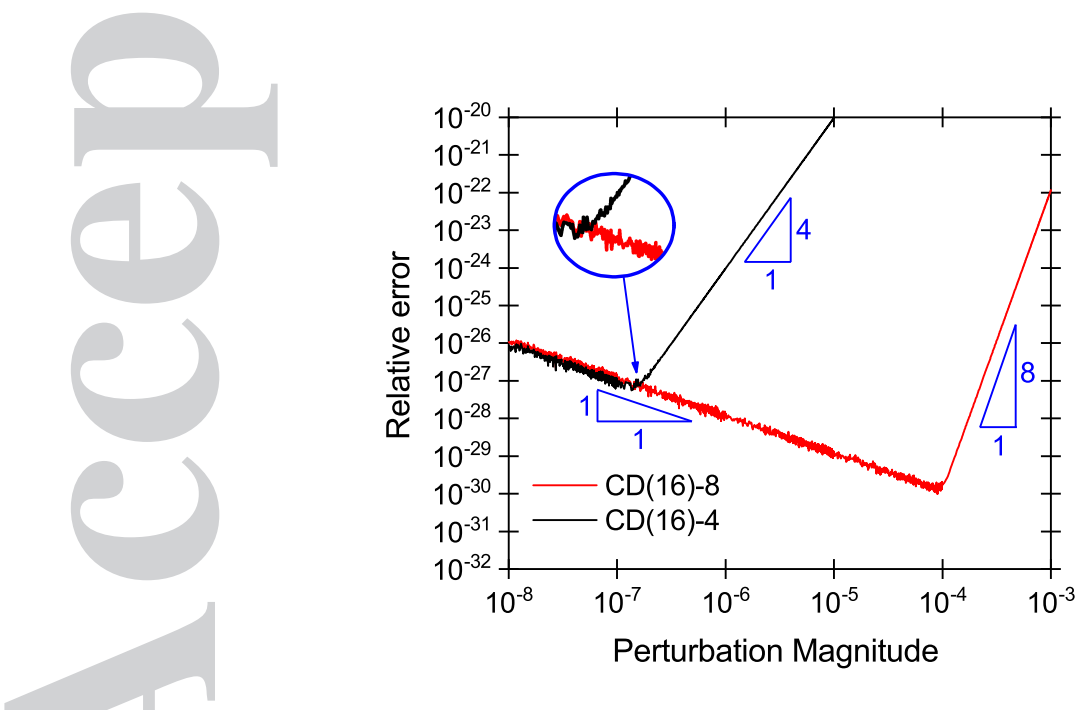

Figure 3. Relative error of fourth- and eighth-order approximations of neo-Hookean spatial elasticity modulus.

This article is protected by copyright. All rights reserved. 


\subsection{Discussion of numerical investigation findings}

The numerical investigations show that higher-order and higher floating-point precision approximations both improve the accuracy of approximated elasticity moduli, in the material and spatial configurations. With increasing order of approximation, the accuracy of the approximated elasticity moduli is generally increased but with diminishing returns. The diminishing returns are due to the requirement for a larger perturbation, which results in invalid perturbation errors and is also likely to be influenced by the rounding errors from the additional coefficients. The use of quadruple precision numerical approximations, novel in this context, is the more significant development. Due to round-off errors in the computation of the double precision analytical solutions, the quadruple precision approximated elasticity moduli are more accurate than these analytical solutions for even a first-order approximation. This level of accuracy with real-domain approximations is not known to have otherwise been accomplished in this context.

It is shown that the higher-order and higher floating-point precision approximation methods are complementary. Utilising both of these methods, approximated elasticity moduli tend towards the quadruple precision analytical moduli. However, finite element solvers use only double precision and hence any improvement in accuracy beyond the double precision limit is inconsequential due

to truncation. Therefore, a first-order quadruple precision approximation is a satisfactory means of obtaining precise elasticity moduli. Though another important factor is the associated computational costs of these methods. This is investigated in the context of the finite element method in the following section.

\section{FINITE ELEMENT INVESTIGATION}

The performance of the approximation methods in finite element simulation is investigated in terms of convergence and computational efficiency. Abaqus/Standard is used, which allows for userimplemented materials through its UMAT interface with subroutines written in Fortran. For finite elasticity, at each iteration, the interface provides the deformation gradient and requires calculation of the Cauchy stress and spatial elasticity tensor in terms of the Jaumann-rate of the Cauchy stress. These tensors are computed in Voigt notation, with indices defined as in the previous section, by the pre-defined arrays STRESS(6) and DDSDDE(6,6) respectively, or STRESS(4) and DDSDDE(4,4) for $2 \mathrm{D}$ problems. For quadruple precision codes, these must be calculated using independently declared quadruple precision arrays, which are then truncated to double precision by equating them to the pre-defined arrays. Higher-order approximations, with standard double precision and the novel quadruple precision method, are investigated by solving the well-known Cook's membrane problem. The measured approximate optimal perturbation magnitudes from Table I are used throughout. The convergence and computational efficiency of the approximation methods are compared to a solution obtained using the equivalent analytical elasticity moduli for seven levels of mesh refinement. The UMAT subroutines used here are available in the dataset[21].

\subsection{Cook's membrane}

The Cook's membrane problem is commonly used in the assessment of finite element procedures. The model is shown with dimensions for the third level of mesh refinement with 2048 elements in Figure 4a. It consists of a tapered membrane where one face is fully fixed and a shearing load is applied at the opposite face. The model is simulated in 3D using plane strain boundary conditions such that its thickness is constant. The body is discretised using hybrid C3D8H elements to enforce incompressibility. For all approximation methods, the model is simulated with a shearing load of 15

$\mathrm{N}$ for both neo-Hookean and Gent constitutive models, with the parameters defined in section 3.1.

\subsection{Convergence with optimal perturbation magnitudes}

All approximation methods with optimal perturbation magnitudes were found to attain convergence. The deformed contour plot showing the shear stress in the 1-2 plane is shown in Figure $4 \mathrm{~b}$ and $4 \mathrm{c}$

This article is protected by copyright. All rights reserved. 


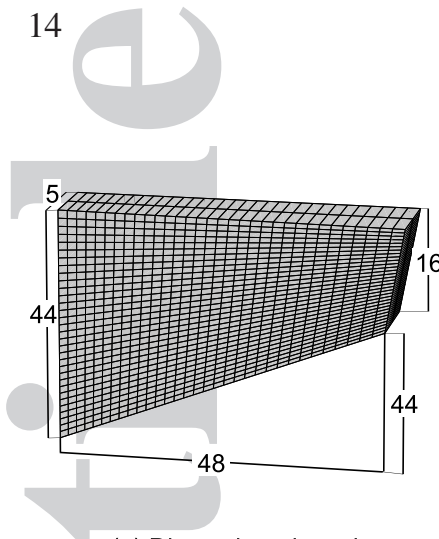

(a) Dimensioned mesh

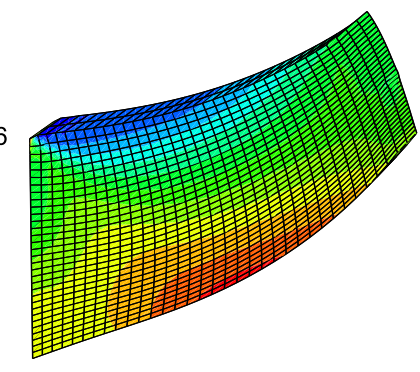

(b) neo-Hookean model

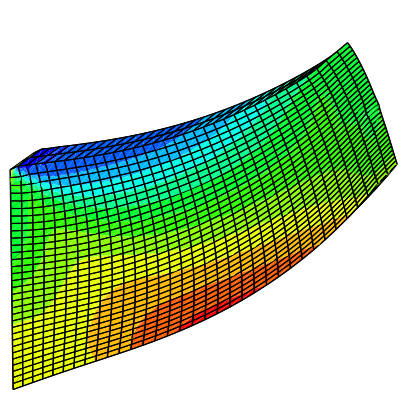

(c) Gent model

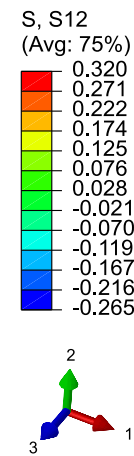

Figure 4. (a) Cook's membrane, dimensions in mm; (b) neo-Hookean, shear stress (MPa); (c) Gent, shear stress (MPa).

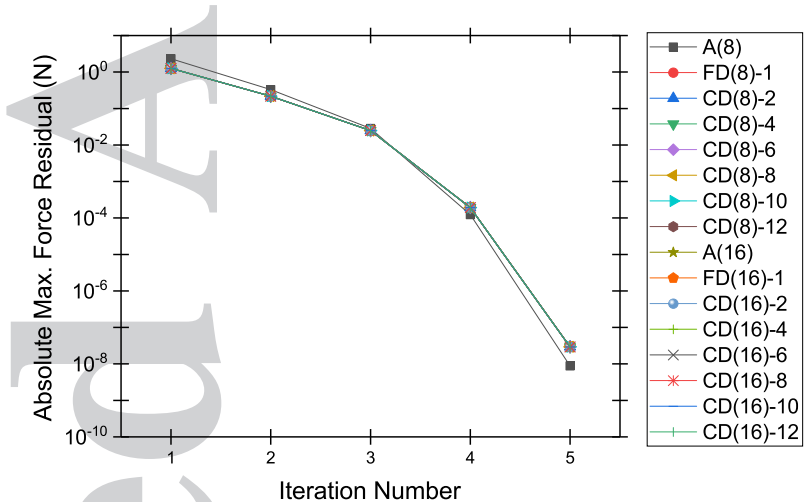

(a) neo-Hookean

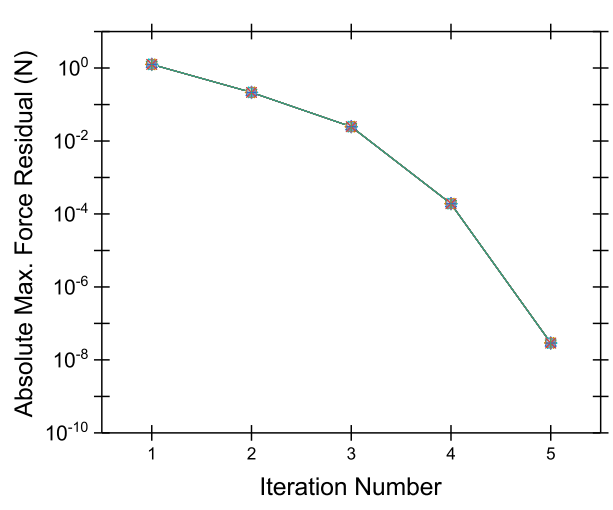

(b) Gent

Figure 5. Maximum force residuals during convergence for (a) neo-Hookean and (b) Gent simulations.

for the neo-Hookean and Gent constitutive models respectively. Given that the stress is computed equivalently for the analytical and approximation methods, the same results are obtained for all measured quantities. Within Abaqus/Standard, the convergence behaviour may be observed by inspection of the maximum residuals in each iteration. The maximum force residuals are plotted for both neo-Hookean and Gent simulations in Figure 5(a) and (b). Both double and quadruple precision analytical elasticity moduli are included, denoted as $\mathrm{A}(8)$ and $\mathrm{A}(16)$ respectively. Though there are some minor differences in the residuals for the different methods, quadratic convergence in five iterations is reliably obtained for all methods.

\subsection{Computational efficiency}

Where available, evaluating the analytical elasticity moduli is more computationally efficient than using numerical approximation. This is due to analytical solutions typically requiring fewer computations and quadratic convergence is generally guaranteed. While the numerical approximations implemented here with optimal perturbation magnitude show quadratic convergence, it is known from the first implementation by Miehe[2] that approximation methods have higher computation times. However, previous studies[1, 2, 15, 18] have shown that with increased mesh refinement the difference between analytical and approximate methods' solve times are decreased. This is due to the increased solution time required for the global matrix iterations in a more complex model, such that the computational effort of the stress and tangent components becomes less significant.

Using seven levels of mesh refinement, the computational efficiency of higher-order approximations, with standard double precision and the novel quadruple precision method, is 

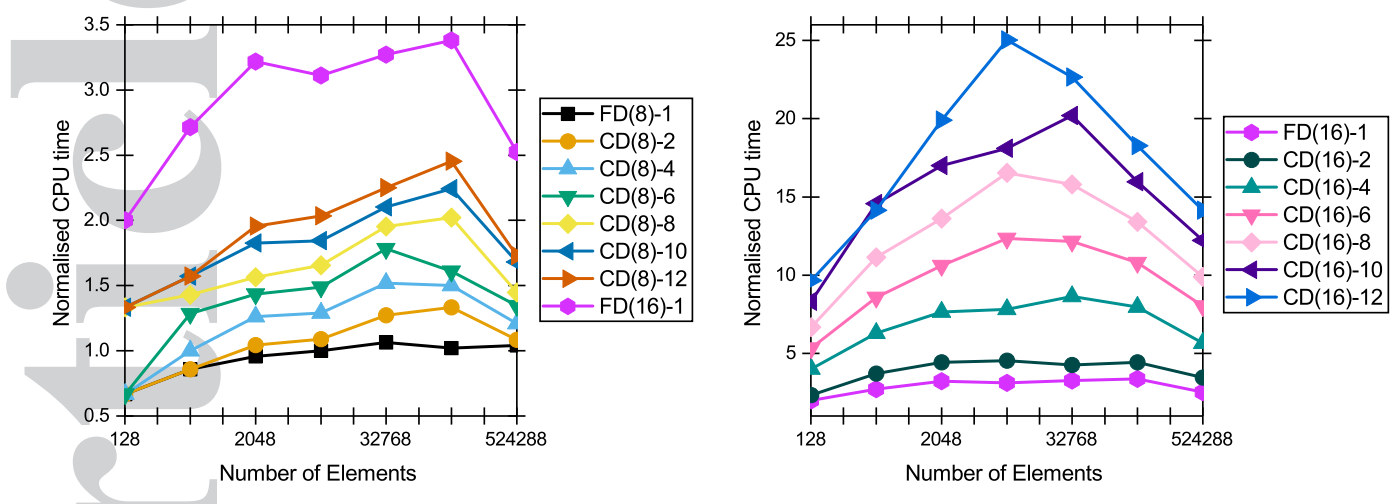

Figure 6. Normalised CPU time for double (left) and quadruple (right) precision approximations.

investigated using the neo-Hookean constitutive model. Mesh refinement is controlled using a constant two elements through the thickness, since stress is constant in this direction. Then, starting with an $8 \times 8$ discretisation, the elements are doubled on each side, increasing the total elements by a factor of four for each subsequent mesh. Since this is a finite elasticity problem, the elasticity moduli require recalculation at each iteration for all integration points. Therefore the total CPU time is used to compare their computational efficiencies.

The results are given in Figure 6 where double and quadruple precision results are separated due to their considerable difference. To demonstrate the disparity, and for improved clarity, the quadruple precision first-order forward difference approximation $(\operatorname{FD}(16)-1)$ result is included in both plots. The plots show that even the first-order forward difference in quadruple precision requires significantly higher CPU times than all orders of double precision approximation. In contrast, the double precision first- and second-order approximations show CPU times similar and even lower than the analytical implementation. Though the lower CPU times are likely due to the somewhat random nature of low duration simulations that was found. Using a greater number of mesh refinements than previous studies[1, 2, 15, 18], the normalised CPU times are shown to generally increase as the mesh is refined until it reaches a maximum before then decreasing with further refinements. However, the quadruple precision approximations all require more than twice the CPU time for all meshes.

\section{CONCLUSIONS}

By investigating the two complementary numerical approximation methods, higher-order and higher floating-point precision numerical approximations, it is shown that elasticity moduli with accuracy comparable to analytical solutions can be obtained using numerical approximations in the realdomain. Using higher-order approximations, accuracy that tends towards the analytical values may be found but with diminishing returns as the order of the approximation increases. However, higher floating-point precision is the more dominant method. By increasing to quadruple precision, even first-order approximations obtain elasticity moduli more accurate than the double precision analytical solution, due to reduced round-off errors during computation with higher floating-point precision. In current finite element solvers, approximations higher than first-order are not required if quadruple precision is used, since additional increases in accuracy are negated upon truncation. While the computational efficiency decreases for higher floating-point precision approximations, the method guarantees equivalent convergence to an analytical solution with a simpler implementation. Where analytical tangent moduli are available they should be preferred as these generally offer the most computationally efficient implementation. Though quadruple precision approximations remain a useful aid to confirm the derivation of the analytical tangent moduli numerically and for debugging their implementation. When analytical tangent moduli are complicated or intractable, the first-order

This article is protected by copyright. All rights reserved. 
quadruple precision approximation method proposed here provides an accurate and effective means of implementation.

\section{ACKNOWLEDGMENTS}

This work was supported in full by an Engineering and Physical Sciences Research Council (EPSRC) grant. Grant number: EP/N509760/1 - studentship 1811648.

\section{DATA STATEMENT}

The Fortran programs and subroutines created during this research are made openly available from the University of Strathclyde "Pure" data archive at http://dx.doi.org/10.15129/b202f1b6-4d3948b8-8778-fb4a2aacc5fd.

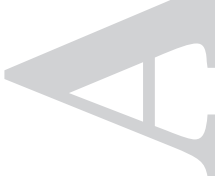

\section{APPENDIX}

\section{A. FINITE DIFFERENCE EQUATIONS AND COEFFICIENTS}

The coefficients for central difference approximations up to the $12^{\text {th }}$-order are calculated using equation (19) and given in Table II.

Table II. Weighted coefficients $c_{m}$ for central difference approximations up to $12^{\text {th }}$-order.

\begin{tabular}{|c|c|c|c|c|c|c|c|c|c|c|c|c|}
\hline & \multicolumn{12}{|c|}{$\mathbf{m}$} \\
\hline$n_{\text {even }}$ & -6 & -5 & -4 & -3 & -2 & -1 & 1 & 2 & 3 & 4 & 5 & 6 \\
\hline 2 & & & & & & $-\frac{1}{2}$ & $\frac{1}{2}$ & & & & & \\
\hline 4 & & & & & $\frac{1}{12}$ & $-\frac{2}{3}$ & $\frac{2}{3}$ & $-\frac{1}{12}$ & & & & \\
\hline 6 & ر & & & $-\frac{1}{60}$ & $\frac{3}{20}$ & $-\frac{3}{4}$ & $\frac{3}{4}$ & $-\frac{3}{20}$ & $\frac{1}{60}$ & & & \\
\hline 8 & 1 & & $\frac{1}{280}$ & $-\frac{4}{105}$ & $\frac{1}{5}$ & $-\frac{4}{5}$ & $\frac{4}{5}$ & $-\frac{1}{5}$ & $\frac{4}{105}$ & $-\frac{1}{280}$ & & \\
\hline 1 & & $-\frac{1}{1260}$ & $\frac{5}{504}$ & $-\frac{5}{84}$ & $\frac{5}{21}$ & $-\frac{5}{6}$ & $\frac{5}{6}$ & $-\frac{5}{21}$ & $\frac{5}{84}$ & $-\frac{5}{504}$ & $\frac{1}{1260}$ & \\
\hline 12 & $\frac{1}{5544}$ & $-\frac{1}{385}$ & $\frac{1}{56}$ & $-\frac{5}{63}$ & $\frac{15}{56}$ & $-\frac{6}{7}$ & $\frac{6}{7}$ & $-\frac{15}{56}$ & $\frac{5}{63}$ & $-\frac{1}{56}$ & $\frac{1}{385}$ & $\frac{1}{5544}$ \\
\hline
\end{tabular}

\section{B. ANALYTICAL ELASTICITY MODULI FOR FIRST INVARIANT HYPERELASTICITY}

The analytical stress and elasticity tensors are defined for any hyperelastic constitutive model defined in terms of the first invariant $\bar{I}_{1}$ of the Cauchy-Green strain tensors and the determinant of the deformation gradient $J$. For both material and spatial definitions, user input of four coefficients $(a, b, c \& d)$ is required, which are defined as follows

$$
a=2 \frac{\partial W}{\partial \overline{\mathrm{I}}_{1}} ; b=4 \frac{\partial^{2} W}{\partial \overline{\mathrm{I}}_{1}^{2}} ; c=\frac{\partial U}{\partial J} ; d=\frac{\partial^{2} U}{\partial J^{2}}
$$

This article is protected by copyright. All rights reserved. 
The implementations are therefore general and require only the definition of these coefficients for all hyperelastic constitutive models defined in terms of $\bar{I}_{1}$ and $J$. For the neo-Hookean and Gent constitutive models defined in section 3.1, these coefficients are given in table III.

Table III. Coefficients for calculation of analytical stress and elasticity moduli.

\begin{tabular}{lccccc}
\hline & $a$ & $b$ & $c$ & $d$ \\
\hline $\begin{array}{l}\text { neo-Hookean } \\
\text { Gent }\end{array}$ & $\frac{2 C_{1}}{\left(1-\frac{I_{1}-3}{J_{m}}\right)}$ & $\frac{2 \mu}{J_{m}} \frac{1}{\left(1-\frac{\bar{I}_{1}-3}{J_{m}}\right)^{2}}$ & $\frac{k_{0}}{2}\left(J-\frac{1}{J}\right)$ & $\frac{k_{0}}{2}\left(1+\frac{1}{J^{2}}\right)$ \\
\hline
\end{tabular}

\section{B.1. Analytical material tensors}

In the material configuration the $2^{\text {nd }}$ Piola-Kirchhoff stress is defined as:

$$
\mathbf{S}=\mathbf{S}_{i s o}+\mathbf{S}_{v o l}=a\left[J^{-2 / 3}\left(\mathbf{1}-\frac{1}{3} I_{1} \mathbf{C}^{-1}\right)\right]+c\left(J \mathbf{C}^{-1}\right)
$$

The material elasticity tensor is defined as

$$
\begin{gathered}
\mathbb{C}=\mathbb{C}_{\text {iso }}+\mathbb{C}_{\text {vol }} \\
\mathbb{C}_{i s o}=\frac{2 a}{3} J^{-\frac{2}{3}}\left[I_{1}\left(\mathbf{C}^{-1} \odot \mathbf{C}^{-1}\right)-\left[\left(\mathbf{C}^{-1} \otimes \mathbf{1}\right)+\left(\mathbf{1} \otimes \mathbf{C}^{-1}\right)\right]+\frac{1}{3} I_{1}\left(\mathbf{C}^{-1} \otimes \mathbf{C}^{-1}\right)\right] \\
+b J^{-\frac{4}{3}}\left[(\mathbf{1} \otimes \mathbf{1})-\frac{1}{3} I_{1}\left[\left(\mathbf{C}^{-1} \otimes \mathbf{1}\right)-\left(\mathbf{1} \otimes \mathbf{C}^{-1}\right)\right]+\frac{1}{9} I_{1}^{2}\left(\mathbf{C}^{-1} \otimes \mathbf{C}^{-1}\right)\right] \\
\mathbb{C}_{v o l}=J(c+d J)\left(\mathbf{C}^{-1} \otimes \mathbf{C}^{-1}\right)-2 c J\left(\mathbf{C}^{-1} \odot \mathbf{C}^{-1}\right)
\end{gathered}
$$

B.2. Analytical spatial tensors

In the spatial configuration, the Kirchhoff stress is defined as

$$
\boldsymbol{\tau}=\boldsymbol{\tau}_{i s o}+\boldsymbol{\tau}_{v o l}=a\left(\overline{\mathbf{b}}-\frac{1}{3} \overline{I_{1}} \mathbf{1}\right)+c(J \mathbf{1})
$$

The spatial elasticity tensor defined in terms of the Oldroyd-rate of the Kirchhoff stress is defined as

$$
\begin{gathered}
={ }_{i s o}+{ }_{v o l} \\
\text { iso }=\frac{2 a}{3}\left[\overline{I_{1}}(\mathbf{1} \odot \mathbf{1})-[(\mathbf{1} \otimes \overline{\mathbf{b}})+(\overline{\mathbf{b}} \otimes \mathbf{1})]+\frac{1}{3} \overline{I_{1}}(\mathbf{1} \otimes \mathbf{1})\right] \\
+b\left[(\overline{\mathbf{b}} \otimes \overline{\mathbf{b}})-\frac{1}{3} \overline{I_{1}}[(\mathbf{1} \otimes \overline{\mathbf{b}})+(\overline{\mathbf{b}} \otimes \mathbf{1})]+\frac{1}{9}{\overline{I_{1}}}^{2}(\mathbf{1} \otimes \mathbf{1})\right] \\
\qquad v o l=J(c+d J)(\mathbf{1} \otimes \mathbf{1})-2 c J(\mathbf{1} \odot \mathbf{1})
\end{gathered}
$$

For finite element codes where the Cauchy stress tensor and spatial elasticity tensor in terms of the Jaumann-rate of the Cauchy stress are required, the Cauchy stress is related to the Kirchhoff stress by $\boldsymbol{\sigma}=J^{-1} \tau$ to give

This article is protected by copyright. All rights reserved. 


$$
\boldsymbol{\sigma}=\boldsymbol{\sigma}_{i s o}+\boldsymbol{\sigma}_{v o l}=a J^{-1}\left(\overline{\mathbf{b}}-\frac{1}{3} \overline{I_{1}} \mathbf{1}\right)+c(\mathbf{1})
$$

The Jaumann-rate elasticity tensor is related to the Oldroyd-rate elasticity tensor as previously defined in (15) to give

$$
\begin{gathered}
A B Q={ }_{A B Q_{i s o}}+{ }_{A B} Q_{v o l} \\
A B Q_{i s o}=a J^{-1}\left[(\mathbf{1} \odot \overline{\mathbf{b}})+(\overline{\mathbf{b}} \odot \mathbf{1})-[(\mathbf{1} \otimes \overline{\mathbf{b}})(\mathbf{1} \otimes \overline{\mathbf{b}})+]+\frac{2}{9} \overline{I_{1}}(\mathbf{1} \otimes \mathbf{1})\right] \\
+b J^{-1}\left[(\overline{\mathbf{b}} \otimes \overline{\mathbf{b}})-\frac{1}{3} \overline{I_{1}}[(\mathbf{1} \otimes \overline{\mathbf{b}})+(\overline{\mathbf{b}} \otimes \mathbf{1})]+\frac{1}{9}{\overline{I_{1}}}^{2}(\mathbf{1} \otimes \mathbf{1})\right] \\
A B Q_{v o l}=(c+d J)(\mathbf{1} \otimes \mathbf{1})
\end{gathered}
$$

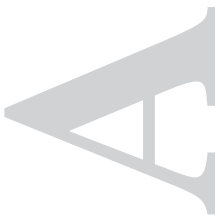

\section{REFERENCES}

1. Tanaka M, Fujikawa M, Balzani D, Schröder J. Robust numerical calculation of tangent moduli at finite strains based on complex-step derivative approximation and its application to localization analysis. Computer Methods in Applied Mechanics and Engineering 2014; 269: 454-470. doi: 10.1016/j.cma.2013.11.005

2. Miehe C. Numerical computation of algorithmic (consistent) tangent moduli in large-strain computational inelasticity. Computer Methods in Applied Mechanics and Engineering 1996; 134(96): 223-240. doi: 10.1016/0045-7825(96)01019-5

3. Young JM, Yao J, Ramasubramanian A, Taber LA, Perucchio R. Automatic Generation of User Material Subroutines for Biomechanical Growth Analysis. Journal of Biomechanical Engineering 2010; 132(10): 104505. doi: $10.1115 / 1.4002375$

4. Korelc J. Multi-language and multi-environment generation of nonlinear finite element codes. Engineering with Computers 2002; 18(4): 312-327. doi: 10.1007/s003660200028

5. Korelc J. Automation of primal and sensitivity analysis of transient coupled problems. Computational Mechanics 2009; 44(5): 631-649. doi: 10.1007/s00466-009-0395-2

6. Rothe S, Hartmann S. Automatic differentiation for stress and consistent tangent computation. Archive of Applied Mechanics 2015; 85(8): 1103-1125. doi: 10.1007/s00419-014-0939-6

7. Wriggers P. Nonlinear Finite Element Methods. Berlin, Heidelberg: Springer Berlin Heidelberg . 2008

8. Sun W, Chaikof EL, Levenston ME. Numerical Approximation of Tangent Moduli for Finite Element Implementations of Nonlinear Hyperelastic Material Models. Journal of Biomechanical Engineering 2008; 130(6): 061003. doi: 10.1115/1.2979872

9. Dennis JE, Schnabel RB. Numerical Methods for Unconstrained Optimization and Nonlinear Equations. 16. Siam 1996.

10. Mathur R. An analytical approach to computing step sizes for finite-difference derivatives. Phd thesis. The University of Texas at Austin, http://hdl.handle.net/2152/ETD-UT-2012-05-5275; 2012.

11. Pérez-Foguet A, Rodríguez-Ferran A, Huerta A. Numerical differentiation for local and global tangent operators in computational plasticity. Computer Methods in Applied Mechanics and Engineering 2000; 189(1): 277-296. doi: 10.1016/S0045-7825(99)00296-0

12. Pérez-Foguet A, Rodríguez-Ferran A, Huerta A. Numerical differentiation for non-trivial consistent tangent matrices: an application to the MRS-Lade model. International Journal for Numerical Methods in Engineering 2000; 48(2): 159-184. doi: 10.1002/(SICI)1097-0207(20000520)48:2;159::AID-NME871 ‘3.0.CO;2-Y

13. Kiran R, Khandelwal K. Complex step derivative approximation for numerical evaluation of tangent moduli. Computers and Structures 2014; 140: 1-13. doi: 10.1016/j.compstruc.2014.04.009

14. Kiran R, Khandelwal K. Numerically approximated Cauchy integral (NACI) for implementation of constitutive models. Finite Elements in Analysis and Design 2014; 89: 33-51. doi: 10.1016/j.finel.2014.05.016

15. Liu H, Sun W. Computational efficiency of numerical approximations of tangent moduli for finite element implementation of a fiber-reinforced hyperelastic material model. Computer Methods in Biomechanics and Biomedical Engineering 2016; 19(11): 1171-1180. doi: 10.1080/10255842.2015.1118467

16. Martins JRRA, Hwang JT. Review and Unification of Methods for Computing Derivatives of Multidisciplinary Computational Models. AIAA Journal 2013; 51(11): 2582-2599. doi: 10.2514/1.J052184

17. Fike J, Alonso J. The Development of Hyper-Dual Numbers for Exact Second-Derivative Calculations. In: . 886. 49th AIAA Aerospace Sciences Meeting including the New Horizons Forum and Aerospace Exposition. American Institute of Aeronautics and Astronautics; 2011; Reston, Virigina: 124

18. Tanaka M, Sasagawa T, Omote R, Fujikawa M, Balzani D, Schröder J. A highly accurate 1st- and 2nd-order differentiation scheme for hyperelastic material models based on hyper-dual numbers. Computer Methods in Applied Mechanics and Engineering 2015; 283: 22-45. doi: 10.1016/j.cma.2014.08.020

This article is protected by copyright. All rights reserved. 
19. Bailey DH, Barrio R, Borwein JM. High-precision computation: Mathematical physics and dynamics. Applied Mathematics and Computation 2012; 218(20): 10106-10121. doi: 10.1016/j.amc.2012.03.087

20. Connolly SJ. Fortran programs and Abaqus subroutines: Higher-order and higher precision numerical approximations of finite strain elasticity moduli. https://www.doi.org/10.15129/b202f1b6-4d39-48b8-8778fb4a2aacc5fd; 2018

21. Holzapfel GA. Nonlinear Solid Mechanics: A Continuum Approach for Engineering. Chichester, UK: Wiley \& Sons, Ltd. . 2000

22. Bažant ZP, Gattu M, Vorel J. Work conjugacy error in commercial finite-element codes: Its magnitude and how to compensate for it. Proceedings of the Royal Society A: Mathematical, Physical and Engineering Sciences 2012; 468(2146): 3047-3058. doi: 10.1098/rspa.2012.0167

23. Simo JC, Hughes T. Computational Inelasticity. 7 of Interdisciplinary Applied Mathematics. New York: SpringerVerlag. 1998

24. Khan IR, Ohba R. New finite difference formulas for numerical differentiation. Journal of Computational and Applied Mathematics 2000; 126(1-2): 269-276. doi: 10.1016/S0377-0427(99)00358-1

25. Li J. General explicit difference formulas for numerical differentiation. Journal of Computational and Applied Mathematics 2005; 183(1): 29-52. doi: 10.1016/j.cam.2004.12.026

26. Fornberg B. Generation of finite difference formulas on arbitrarily spaced grids. Mathematics of Computation 1988; 51(184): 699-699. doi: 10.1090/S0025-5718-1988-0935077-0

27. Isaacson E, Keller HB. Analysis of numerical methods. Courier Corporation. 1994

28. Miehe C, Göktepe S. A micro-macro approach to rubber-like materials. Part II: The micro-sphere model of finite rubber viscoelasticity. Journal of the Mechanics and Physics of Solids 2005; 53(10): 2231-2258. doi: 10.1016/j.jmps.2005.04.006

29. Hossain M, Vu DK, Steinmann P. Experimental study and numerical modelling of VHB 4910 polymer. Computational Materials Science 2012; 59: 65-74. doi: 10.1016/j.commatsci.2012.02.027

30. Liao Z, Yao X, Zhang L, Hossain M, Wang J, Zang S. Temperature and strain rate dependent large tensile deformation and tensile failure behavior of transparent polyurethane at intermediate strain rates. International Journal of Impact Engineering 2019; 129(January): 152-167. doi: 10.1016/j.ijimpeng.2019.03.005

31. Simo JC, Taylor RL. Consistent tangent operators for rate-independent elastoplasticity. Computer Methods in Applied Mechanics and Engineering 1985; 48(1): 101-118. doi: 10.1016/0045-7825(85)90070-2

32. Ogden RW, Roxburgh DG. A pseudo-elastic model for the Mullins effect in filled rubber. Proceedings of the Royal Society A: Mathematical, Physical and Engineering Sciences 1999; 455(1988): 2861-2877. doi: 10.1098/rspa.1999.0431

33. Göktepe S, Miehe C. A micro-macro approach to rubber-like materials. Part III: The micro-sphere model of anisotropic Mullins-type damage. Journal of the Mechanics and Physics of Solids 2005; 53(10): 2259-2283. doi: 10.1016/j.jmps.2005.04.010

34. Miehe C, Keck J. Superimposed finite elastic-viscoelastic-plastoelastic stress response with damage in filled rubbery polymers. Experiments, modelling and algorithmic implementation. Journal of the Mechanics and Physics of Solids 2000; 48(2): 323-365. doi: 10.1016/S0022-5096(99)00017-4

35. Wang S, Chester SA. Experimental characterization and continuum modeling of inelasticity in filled rubber-like materials. International Journal of Solids and Structures 2018; 136-137: 125-136. doi: 10.1016/j.ijsolstr.2017.12.010

36. Rivlin RS. Large Elastic Deformations of Isotropic Materials. I. Fundamental Concepts. PhilosophicalTransactions of the Royal Society A: Mathematical, Physical and Engineering Sciences 1948; 240(822): 459-490. doi: 10.1098/rsta.1948.0002

37. Gent AN. A New Constitutive Relation for Rubber. Rubber Chemistry and Technology 1996; 69(1): 59-61. doi: $10.5254 / 1.3538357$

38. Hartmann S, Gilbert RR. Identifiability of material parameters in solid mechanics. Archive of Applied Mechanics 2017; 88(1): 1-24. doi: 10.1007/s00419-017-1259-4

39. Steinmann P, Hossain M, Possart G. Hyperelastic models for rubber-like materials: Consistent tangent operators and suitability for Treloar's data. Archive of Applied Mechanics 2012; 82(9): 1183-1217. doi: 10.1007/s00419012-0610-z

40. Hossain M, Steinmann P. More hyperelastic models for rubber-like materials: consistent tangent operators and comparative study. Journal of the Mechanical Behavior of Materials 2013; 22(1-2): 27-50. doi: 10.1515/jmbm2012-0007

41. Kaliske M, Rothert H. On the finite element implementation of rubber?like materials at finite strains. Engineering Computations 1997; 14(2): 216-232. doi: 10.1108/02644409710166190

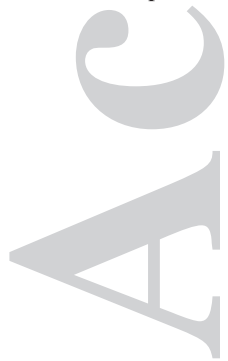

This article is protected by copyright. All rights reserved. 